\title{
Benefits of Selective Packet Discard in Networks-on-Chip
}

\author{
ANDREAS LANKES, THOMAS WILD, STEFAN WALLENTOWITZ, and ANDREAS \\ HERKERSDORF, Technische Universitaet Muenchen, Institute for Integrated Systems
}

Today, Network on Chip concepts principally assume inherent lossless operation. Considering that future nanometer CMOS technologies will witness increased sensitivity to all forms of manufacturing and environmental variations (e.g., IR drop, soft errors due to radiation, transient temperature induced timing problems, device aging), efforts to cope with data corruption or packet loss will be unavoidable. Possible counter measures against packet loss are the extension of flits with ECC or the introduction of error detection with retransmission.

We propose to make use of the perceived deficiency of packet loss as a feature. By selectively discarding stuck packets in the NoC, a proven practice in computer networks, all types of deadlocks can be resolved. This is especially advantageous for solving the problem of message-dependent deadlocks, which otherwise leads to high costs either in terms of throughput or chip area. Strict ordering, the most popular approach to this problem, results in a significant buffer overhead and a more complex router architecture. In addition, we will show that eliminating local network congestions by selectively discarding individual packets also can improve the effective throughput of the network. The end-to-end retransmission mechanism required for the reliable communication, then also provides lossless communication for the cores.

Categories and Subject Descriptors: C.1.2 [Processor Architectures]: Multiple Data Stream Architectures (Multiprocessors)—Interconnection architectures; C.1.4 [Processor Architectures]: Parallel Architectures-Distributed architectures

General Terms: Design, Performance, Reliability

Additional Key Words and Phrases: Network-on-chip, message dependent deadlocks, lossy, selective discard, retransmission, reliable communication, deadlock avoidance, strict ordering

\section{ACM Reference Format:}

Lankes, A., Wild, T., Wallentowitz, S., and Herkersdorf, A. 2012. Benefits of selective packet discard in networks-on-chip. ACM Trans. Architec. Code Optim. 9, 2, Article 12 (June 2012), 21 pages.

DOI $=10.1145 / 2207222.2207228$ http://doi.acm.org/10.1145/2207222.2207228

\section{INTRODUCTION}

The progress in process technology has enabled the integration of a steadily growing number of transistors on one chip. In consequence, more and more components can be integrated on a single chip. This continuing growth of functionality in todays SoCs has already lead to the requirement of a new on-chip communication paradigm: Networkson-chip (NoCs) have been proposed [Bjerregaard and Mahadevan 2006; Jantsch and et al. 2003; Benini and Micheli 2002] as communication infrastructures for SoCs that require scalability, since shared buses can no longer cope with the also rising communication bandwidth requirements.

Author's address: A. Lankes; email: andreas.lankes@tum.de.

Permission to make digital or hard copies of part or all of this work for personal or classroom use is granted without fee provided that copies are not made or distributed for profit or commercial advantage and that copies show this notice on the first page or initial screen of a display along with the full citation. Copyrights for components of this work owned by others than ACM must be honored. Abstracting with credit is permitted. To copy otherwise, to republish, to post on servers, to redistribute to lists, or to use any component of this work in other works requires prior specific permission and/or a fee. Permissions may be requested from Publications Dept., ACM, Inc., 2 Penn Plaza, Suite 701, New York, NY 10121-0701 USA, fax +1 (212) 869-0481, or permissions@acm.org.

(c) 2012 ACM 1544-3566/2012/06-ART12 $\$ 10.00$

DOI 10.1145/2207222.2207228 http://doi.acm.org/10.1145/2207222.2207228 

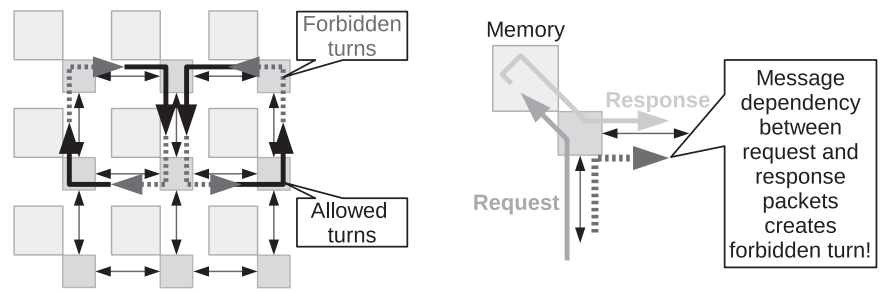

Fig. 1. Message dependencies in a network using XY-routing.

However, the constant shrinking of feature sizes in new process technologies also poses problems, especially in the field of reliability [Borkar 2005; Mitra et al. 2005; Murali et al. 2005; Borkar 2010]. While geometrical sizes of chips and thus link lengths do not scale, the wire width is decreasing with feature sizes. In consequence, increasing wire resistance and coupling capacitance lead to a higher probability of bit transmission errors. Thus, on-chip transmission becomes more and more vulnerable to transient errors. In addition, storage of data in registers and memories also becomes more susceptible, since logic states are represented by constantly shrinking charges. This not only affects the processing cores in SoCs, but also the on-chip network, which consists of routers and network adapters.

To guarantee reliable communication in the presence of transient errors different approaches have already been proposed [Kang et al. 2010; Park et al. 2006; Murali et al. 2005]. These networks either add error correction codes (ECC) to the packets or rely on cyclic redundancy checks (CRC) of the transferred data and retransmission in case an error is detected. The problem with ECC based concepts is that it also requires hardening the router logic against any kind of soft errors. Although, this could be done (e.g., with triple modular redundancy [Mitra et al. 2005]), it results in high hardware overhead. In contrast, retransmission based approaches can also tolerate transient errors in the router's functionality, and thus will become unavoidable. Retransmission can either be realized as end-to-end retransmission of packets or as proposed by most papers on hop-by-hop retransmission of flits.

In addition to these reliability issues, packet switched NoCs, especially networks based on wormhole forwarding, suffer from deadlocks. Deadlocks originating from routing cycles in the network can easily be avoided by ensuring acyclic network paths [Dally and Seitz 1987]. This can be realized by restrictions of the routing functions or by the implementation of additional channels and the adaptation of the routing function. However, in a NoC deadlocks can also arise from message-dependencies [Hannsson et al. 2007] occurring in cores, even if the network itself is free of routing cycles. Figure 1 shows how the dependency between request and response at a memory implicitly creates a turn, which actually was forbidden to prevent routing cycles. A variety of approaches has been proposed to cope with message dependent deadlocks: a number of different methods classified as deadlock avoidance [Hannsson et al. 2007] mechanisms, and also deadlock recovery [Lankes et al. 2010] that resolves occurring deadlocks during runtime. Examples of such deadlock avoidance mechanisms are strict ordering or end-to-end flow control. However, all these solutions to message-dependent deadlocks have to be paid either by decreased network performance or by additional buffer-space and thus increased chip area.

In our opinion, trying to keep NoCs strictly lossless while solving the problems of message-dependent deadlocks and reliable communication leads to overengineered networks. In order to approach these problems we propose to drop the popular paradigm of lossless communication in NoCs. Instead we propose sdNoC, a NoC that actively 
performs a selective discard of packets not only in case of bit errors but also to resolve message-dependent deadlocks. As this solution does not require virtual channels compared to strict ordering, it allows for a simpler router architecture, faster arbitration and for better adaptability in regard to chip area and network throughput. If lower network throughput is sufficient, the $\mathrm{sdNoC}$ routers can operate with half the buffer space compared to routers implementing strict ordering (assuming two dependent message types in the system). The buffer requirements of strict ordering can also be reduced by dynamic sharing of queues, but this introduces more complex control in the routers [Su et al. 2011]. The throughput of sdNoC can be increased by extending the length of the router queues, while still keeping the advantage of a simpler router architecture. Another advantage of sdNoC is that it inherently supports all communication protocols independent of the number of dependent messages. In contrast, the number of virtual channels in a NoC based on strict ordering has to be carefully adapted to the number of dependent messages in the communication protocols that later are used in the system.

By backing up this bounded loss of sdNoC with end-to-end retransmission capabilities, reliable communication can be guaranteed and all types of deadlocks can be handled with a minimum of additional cost. The approach of selectively discarding packets is not very popular in the field of NoCs. Especially, in the context of message-dependent deadlocks limited packet loss in NoCs has not been proposed as a solution before. In contrast, many communication networks and the protocols are designed to cope with packet loss. For example the TCP protocol secures the communication against packet loss and thus provides reliable communication. In general, this loss is not caused by unreliable communication media or by buffer overflow. It is even introduced on purpose by the early random discard policy applied in network processors. Hereby, single packets are dropped in order to increase the throughput for the traffic in total.

The rest of the article is structured as follows: Section 2 presents related work in the field of fault-tolerant NoCs and message-dependent deadlocks. In Section 3 we describe our concept sdNoC in more detail and Section 4 presents a complexity evaluation of the compared concepts. Section 5 shows the investigation of our approach in terms of performance. Section 6 concludes this article.

\section{RELATED WORK}

Deadlocks and fault tolerance have already been the topic of research in the field of parallel computer networks. Kim et al. [1994] propose a concept named Compressionless Routing that uses its retransmission buffers to handle deadlocks and provide fault tolerance at the same time. Since Compressionless Routing implements adaptive routing in wormhole based torus networks without virtual channels, deadlock situations can occur in the network. Although these situations are quite rare according Kim et al. [1994], the network must be able to handle them. In their concept the sender's network adapter detects the deadlock of a sent packet and then withdraws the packet from the network. This means, a packet may only lose the connection to its sender after its header flit has entered the destination tile, and thus can no longer become part of a deadlock. If a packet is not long enough to form a channel from the sender to destination, then packets are extended by adding pad flits. A withdrawn packet, is retransmitted after a certain delay from a retransmission buffer. The method of padding packets leads to significant overhead. According to the authors much lower throughput is observed in large networks for packets of less than 32 flits in size. In Fault-tolerant Compressionless Routing proposed by Kim et al. [1994], which provides tolerance towards transient errors, the padding of packets is even continued until the actual tail flit of the packet has entered the destination. This additional overhead decreases the network throughput even further (30\%). 
In the field of NoCs, one of the few published concepts that implement lossy transport is the Proteo NoC [Saastamoinen et al. 2003]. In Proteo routers drop packets in case of buffer overflow and are based on a hop-by-hop retransmission scheme. However, in case of a loaded network or temporary local congestion this leads to a high number of dropped packets. Besides, Saastamoinen et al. [2003] did not provide an investigation or evaluation of the lossy concept for NoCs in terms of throughput or regarding its ability to solve message dependent deadlocks.

Apart from Proteo there is the class of bufferless NoCs [Gomez et al. 2008; Hayenga et al. 2009; Moscibroda and Mutlu 2009; Fallin et al. 2011] that in part also handle deadlocks by dropping packets. The concept of bufferless NoCs tries to avoid buffers in the routers, since they are the main contributors to power consumption [Hoskote et al. 2007] and chip area requirements [Gratz et al. 2006]. By using only registers in the routers, higher router clock rates can be achieved [Gomez et al. 2008]. However, packets always have to move on with the next clock cycle. If two packets in a router contend for the same output port, one packet either has to be misrouted or dropped. If misrouting is allowed in a network, the problem of livelocks has to be considered.

The routers of the BPS network architecture [Gomez et al. 2008] can drop packets and request a retransmission from the resend buffer of the source by sending a NACK packet. However, if packets were dropped in every case of output port contention in a router, this would lead to very high drop rates. Thus, in order to decrease the drop rate, packets are first misrouted in Gomez et al. [2008] and the network is overprovisioned by using SDM (space division multiplexing) with very wide links (256 bit). Since NACK packets must not be dropped, the routers in BPS require small buffers in which these packets can be stored if they have to be dropped from the network. SCARAB [Hayenga et al. 2009] a similar bufferless network concept removes the requirement for the small NACK buffers by sending NACKs over a circuit switched network.

Since BLESS [Moscibroda and Mutlu 2009] does not have retransmission buffers, packets cannot be dropped, only be misrouted. In order to avoid deadlocks, all flits of a packet store the header information to allow different routes for the flits of a packet if necessary. As flits of a packet can enter the destination out of order, reassembly buffers are required. When sizing these buffers reassembly deadlocks [Fallin et al. 2011] have to be taken into account. To avoid this, infinitely large reassembly buffers were assumed in Moscibroda and Mutlu [2009]. The network concept CHIPPER presented in Fallin et al. [2011] avoids the worst case sizing of the reassembly buffers by allowing the discard of packets in certain situations.

While bufferless network concepts also solve the problem of deadlocks, their peak network throughput is lower than that of buffered networks. According to Fallin et al. [2011] bufferless NoCs are preferably used for low or medium loaded networks. However, around hotspots in a network similar conditions as in a highly loaded network can be observed. In contrast to the resend buffers used in Gomez et al. [2008] or Hayenga et al. [2009], which could possibly be used for providing fault tolerance via retransmission, reassembly buffers as used in Moscibroda and Mutlu [2009] or Fallin et al. [2011] cannot be used for this matter.

The given solutions to deadlocks do not require additional measures to handle message-dependent deadlocks since they solve deadlocks in general. However, the most popular approach to prevent routing-dependent deadlocks in buffered NoCs is the use of restricted routing functions like XY-routing or turn model based routing. But this approach requires additional measures to handle message-dependent deadlocks. A variety of solutions to counter this problem have been published, most of them avoid the occurrence of deadlocks at runtime. An example of such a deadlock avoidance technique is strict ordering in which packets of different message types (read request, read response) are either separated into different physical [Wentzlaff et al. 2007] or logical 
networks [Gebremichael-Tesfagiorgis 2005]. However, implementing 2 or even more independent networks on a SoC requires significant chip area. If independent networks are realized by virtual channels, buffer requirements can be reduced by sharing buffer space within the router [Liu and Delgado-Frias 2006; Neishaburi and Zilic 2009; Lai et al. 2008]. Sharing of buffers can either be done between virtual channels of one port or even between all ports of the router. This concept can increase the throughput of routers in terms of flits per cycle. However, as Su et al. [2011] show, it also results in significant hardware overhead and thus reduced maximum clock frequency.

Another approach to avoid message-dependent deadlocks are end-to-end flow control mechanisms [Goossens et al. 2005; Durand and Lattard 2005] that assure that there is enough buffer space at the receiver side for each packet that is sent into the network. This requires a mechanism to distribute information on free buffer space from the receiver to the senders. Such a mechanism can be realized with a credit-based distribution scheme. However, this requires [Hannsson et al. 2007] extra buffering to hide the round-trip time of the credits and additional bandwidth is required for the credit communication. In case such credits should be avoided dedicated buffers per communication connection have to be instantiated in the network interface.

Buffer spacing is another deadlock avoidance approach that is widely used in parallel computers [Song and Pinkston 2003]. It is usually not used in NoCs (except Tilera's Tile 64 [Wentzlaff et al. 2007]) due to its large buffer requirements. It guarantees that all packets sent into the network can be received at the destination, by instantiating accordingly large input buffers at the receivers. While storing packets for retransmission at the sender also results in large buffers, these buffers can be used for providing fault tolerance at the same time.

An alternative to deadlock avoidance is deadlock recovery, which allows the occurrence of deadlocks at runtime, but is able to resolve deadlocks and return the network into an operable state again. A deadlock recovery scheme for NoCs has been proposed in Lankes et al. [2010].

All the concepts to counter message-dependent deadlocks already mentioned solve this with different design targets. Some try to minimize the additional implementation cost, while others try to optimize the network performance or enable easy implementation. However, all these approaches, either using deadlock avoidance or recovery, cannot provide a fault tolerant network. For providing reliable communication in the presence of transient errors, the networks already equipped with costly or performance restricting measures to avoid message dependent deadlocks, have to be equipped with additional mechanisms to provide reliable communication. Solutions to guarantee reliable communication in presence of transient errors in on-chip networks have already been presented in the past. Most of these publications propose hop-by-hop based retransmission to secure the transfers on the links against bit flips. [Kang et al. 2010] presents a hardware implementation of such a hop-by-hop based retransmission approach and evaluates it in terms of latency and required chip area overhead. Similarly, in Park et al. [2006] a hop-by-hop based retransmission concept is described and investigated with a simulation model. However, it is only able to cope with bit flips on the links but not able to handle bit flips occurring in the router internal buffers. Murali et al. [2005] compare an end-to-end retransmission scheme with hop-by-hop retransmission in terms of energy efficiency. While hop-by-hop based retransmission shows lower transfer latencies, it has higher buffer space and energy requirements than end-to-end retransmission.

Except Park et al. [2006] none of the fault-tolerant NoC concepts mentioned previously use their additional resources to approach also the problem of deadlocks. However, this concept is only able to handle deadlocks caused by routing cycles. The authors do not address the problem of message-dependent deadlocks and the mechanisms that 
would be required in the network adapters to cope with these deadlocks. In this article we present a method that is able to cope with message-dependencies as well as providing a network that is tolerant of transient errors on the links or in the routers.

\section{SDNOC: SELECTIVE PACKET DISCARD IN NETWORKS-ON-CHIP}

\subsection{Overview}

In many communication networks protocols are designed in a way to handle bounded packet loss, even if the used communication media are very reliable. In fact, the dropping of single packets is done actively in the queues of network equipment (Random early discard, RED [Floyd and Jacobson 1993]) in order to avoid congestion and thus improve the network throughput in total. We propose to apply this idea of actively dropping packets under specific circumstances to on-chip networks. Instead of avoiding deadlocks or redirecting deadlocked packets into reserved channels, we solve deadlocks by simply dropping single packets. A timer based criterion as discard policy even allows to resolve local congestions and to increase the effective network throughput. Together with an end-to-end retransmission scheme the communication infrastructure provides lossless communication on a higher layer and can even provide reliable communication in case of transient errors.

\subsection{Packet Discard and Error Detection}

Deadlocks are a severe problem in packet-switched on-chip networks, which render a network completely useless if they occur and cannot be resolved at runtime. As shown in Section 2, especially the solutions to counter message dependent deadlocks require high efforts. In general, a deadlock in a network is caused by multiple packets that try to enter the next buffer queues along their path, but can not advance as they block each other by forming a circular path. This means each packet waits for another packet to free resources, which will never happen due to a circular dependency. Therefore, we propose to drop one or more packets that are caught in the deadlock and thus free resources for the other packets. Hereby, the deadlock is resolved.

The criterion for dropping a packet is based on the queue latency and realized by a timer. If the header flit of a packet stays in the input or output queue longer than a defined threshold time, the packet is dropped. Adequate values for this threshold are presented in Section 5. This criterion for dropping packets improves the throughput of the network by solving temporary blockages. However, even more important is that this criterion also initiates the dropping of packets that are stuck in a deadlock, since deadlocks result in a infinitely long waiting state.

Dropping a packet in a router queue is started by deleting the header flit and all subsequent flits of this packet in the current queue. In case there still remain flits of this packet in upstream router queues, the deletion command stays active, and on entrance to this queue the remaining flits of the packet are also deleted. The dropping is completed with the deletion of the tail flit. An alternative of this solution would be to distribute the drop command among the queues that store a packet. However, since a packet can not only span over the queues of one router, but also over multiple routers, implementing this would become very complex.

The approach of dropping a limited number of packets is used in many communication networks. An example is the RED policy [Floyd and Jacobson 1993] that discards packets in buffer queues of network processors. However, this policy is not applicable to NoCs, since RED connects the probability to discard packets with the fill level of the queue. Since buffer queues in NoCs are only a few flits long and thus are filled up immediately if there is only a local congestion, this would lead to too high drop rates. 


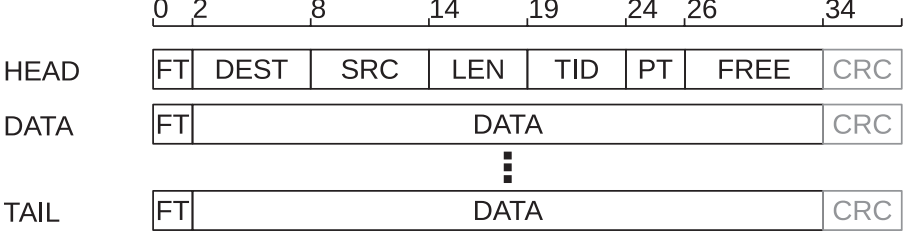

Fig. 2. Packet and flit format of the sdNoc.
FT: Flit type, DEST: Destination, SRC: Source, LEN: Packet length, TID: Transfer id, PT: Packet type, CRC: cylic redundancy check

Another criterion for dropping packets are bit flips. In order to detect these bit flips, we propose to extend the flits by CRC bits. In addition, to detect errors in the packet compound of the flits, the header flit contains the length of the packet (see Figure 2), that is, the number of flits. The error detection is only implemented in the network interface of the tiles. Here, the packet first has to be received completely, checked for errors and then can be dropped in case of an error. In contrast, the routers are not equipped with error detection, since the packets cannot be dropped in the routers of the network but only when they have reached their destination tile. Assuming there would be an error detection unit at the input queue of each router which could detect errors, the packet could be dropped only if the error was detected for the header flit. Other than that, if an error was detected for a data flit or the tail flit, the header flit is most probably already in another queue or even in a downstream router. In this case it is impossible to drop the complete packet.

Although an error detection is not required in the routers, the network must be enabled to handle bit flips in the flit type field (FT = \{head, data, tail $\}$; see Figure 2) of the flits and the destination field of the header flit. Errors in the destination field can simply be solved by using the local output port as default value in the routing logic. In this way, packets with corrupted destination address will leave the network immediately, and be dropped in the network interface. To provide tolerance of the network against bit flips in the flit type field, the routers must implement two rules, apart from their normal wormhole routing functionality.

- R1. After a tail flit, delete all flits until a new header flit arrives. This rule solves the problem of header flits that become data flits $(H \rightarrow D)$ because of a bit flip. The rule makes sure that the complete packet of the corrupted header flit is dropped by the router. Besides, this rule also handles bit flips of type $\mathrm{H} \rightarrow \mathrm{T}$ and $\mathrm{D} \rightarrow \mathrm{T}$.

$-\mathrm{R} 2$. Create a new route for every header flit (even if there was no tail flit before). This rule sees to it that after a tail flit that got corrupted and now is a data flit $(\mathrm{T} \rightarrow \mathrm{D})$, the router nevertheless sets the correct route for the following packet. Rule 2 also handles flit type corruptions of type $\mathrm{T} \rightarrow \mathrm{H}$ and $\mathrm{D} \rightarrow \mathrm{H}$.

These two rules guarantee the operation of the routers for all possible type corruption of the flits. The routers either drop parts of corrupted packets immediately, or route them to a network interface that can detect the corruption of a packet and drop it. With the length field in the header flit, a network interface can also detect an incomplete packet because of a $\mathrm{D} \rightarrow \mathrm{H}$ type flip, in which the second part of this packet was routed to another destination.

This approach secures the communication against all transient bit errors in the routers and links and also against any transient errors in the router logic. In contrast, most hop-by-hop retransmission or ECC schemes cannot handle soft errors in the router functionality, but only secure against transient faults on the link. However, as stated in Frantz et al. [2007] the share of errors occurring in the router is not negligible compared to errors on the links. Although, this way of handling bit transmission errors leads to 
higher traffic compared to a solution with error detection in each router, it decreases the router complexity and thus allows simpler router architecture and possibly higher clock rates. In addition, it significantly reduces the number of required error detection units as compared to a network architecture requiring one detection unit per input port.

\subsection{Retransmission and Transmission Control}

In order to still provide lossless communication, a retransmission mechanism has to be implemented in the network interface. Therefore sent packets have to be stored in a retransmission buffer at the sender tile, to enable the retransmission in case the packet is dropped. This retransmission mechanism is triggered by a timer. Consequently, an individual timer for each sent packet is required and the timeout has to be chosen carefully in order to not resend packets that have not been dropped but have just not arrived yet due to short temporary blockages. Adding a small number of randomly determined cycles to the static retransmission timer, avoids livelock in the drop-retransmit based network probabilistically. While the absence of livelocks is not guaranteed, the probability of livelock occurrence is insignificant. In the simulations livelocks did not occur.

The size of the retransmission buffer directly affects the number of outstanding requests. That is the number of packets that a tile can send into the network, before it has to wait for a transaction to be completed, so that a packet can be removed from the retransmission buffer. In a system consisting of many cores, there exists an optimum number of outstanding requests regarding the total network throughput. A too low number of outstanding requests prevents an efficient operation of the core, while too many outstanding requests also affect the effective throughput negatively. This issue is discussed in Section 4 in more detail. In case the output or sending buffer of a tile can store more packets than the optimum number of outstanding requests, then windowing has to be applied on the outgoing packets in order to not exceed the optimum number of packets.

In order to reduce the number of packets that have to be stored for a possible retransmission, it is only applied to packets that are on the first position in a communication protocol (n-way protocol). In case of a read access to a memory with a read request and a read reply (2-way protocol) only the read request is stored in the retransmission buffer by the sender, so that it can be retransmitted in case one of the packets of this read access is dropped. This requires that the data sent with the read reply, can be sent again if the packet is dropped and it is not overwritten in the meantime.

The advantage of this approach is that a slave, like a memory to which many cores of a $\mathrm{SoC}$ send requests, does not have to store all the answer packets for retransmission. If it would have to do this, the according retransmission buffer would be very large or the sending bandwidth would be limited. In addition, the concept of covering only the first packet in a communication protocol with retransmission capabilities reduces the additional traffic caused by the acknowledgement packets. The alternative would be to send an acknowledgment packet for each transmitted packet.

However, this approach does not work for all kinds of communication partners. Since the answer packet of a slave is not covered by retransmission in this concept, it must be guaranteed that the data can be sent again, if the original request is received again by the slave. In memories this coherence problem must be solved by a superior protocol layer, which makes sure that such kind of data is not overwritten by other processes. For accesses to IO interfaces this method is not applicable. Here, all packets that are part of the communication, request, and response, must be stored for retransmission and thus also be acknowledged. In addition, to avoid coherence problems, functionality to detect duplicate receives has to be implemented in network adapters. This can be done using the transfer and source id shown in Figure 2. 
a)

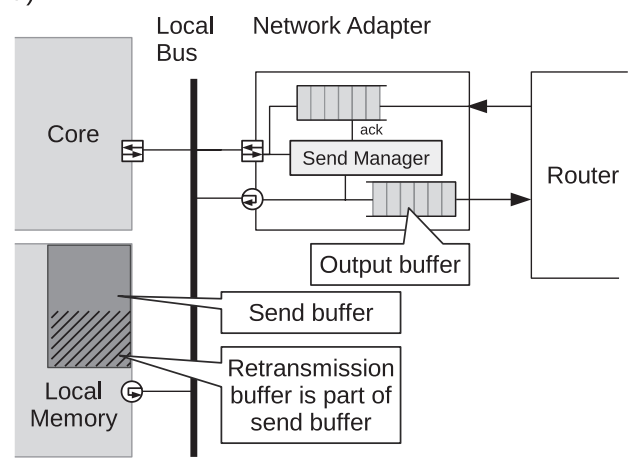

b) Input buffer

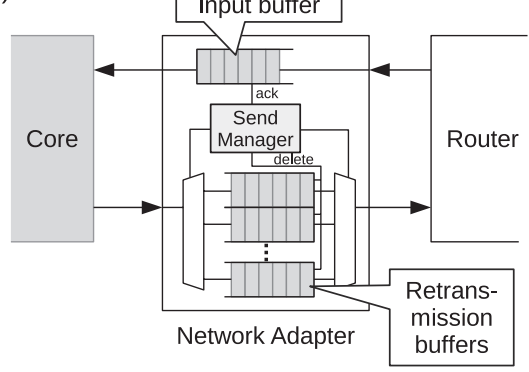

Fig. 3. Implementation of the retransmission buffer in a local memory a) or in the network interface b).

\subsection{Storage Requirements}

The storage required to save packets at the original sender until their successful transfer has been acknowledged, can be realized in two different ways depending on the architecture of the SoC. In our preferred architecture, shown in Figure 3(a), some space of the local memory of a SoC's compute tile is reserved to store sent packets until their acknowledgement. Then, the network interface just requires a sending unit that manages the saving and deleting of packets in the reserved space of the local memory. Besides this, the network interface just needs a conventional output buffer, which stores the flits of the packet that is currently sent out, in order to avoid single accesses to the memory for each flit of a packet. Assuming the retransmission buffer is realized in a local memory of a processor core, the sending buffer should be larger than the optimum number of outstanding requests in order to avoid the processor core having to stall while waiting for a free space in the retransmission buffer. However, then the windowing method has to be applied to restrict the outgoing packets.

The alternative solution for the realization of the retransmission buffer is required, if the tiles of a SoC do not have local memory available for storing the packets that possibly have to be retransmitted. Then the retransmission buffer has to be realized by dedicated buffers in the network interface (see Figure 3(b)). The size of these buffers will most likely be directly chosen according to the optimum number of outstanding requests or even below in order to save chip area.

Independent of how the retransmit buffer is realized, the stored packets have to be protected with ECC. To secure the transfer in the network, a CRC code, which is checked in the destination, is sufficient.

\subsection{Acknowledgments}

A transfer is completed when it has been acknowledged by a confirmation packet. This can either be done explicitly by an acknowledgement packet for instance, for a write request. For read requests the confirmation is done implicitly by the read reply. On reception of such an acknowledgment packet the original packet of this transfer can be removed from the retransmission buffer and the freed space can be used for a new transfer.

The relation between the original packet and the acknowledgement packet is realized with a transfer id in the header flit of the packets. Since this id only has to be unique for the currently active transfers of one tile, that is, its outstanding requests, a field of 4 bits is sufficient for the transfer id. Assuming a quite high number of 10 outstanding requests (see Section 5), a 4 bit transfer id does still not lead to the situation that just 

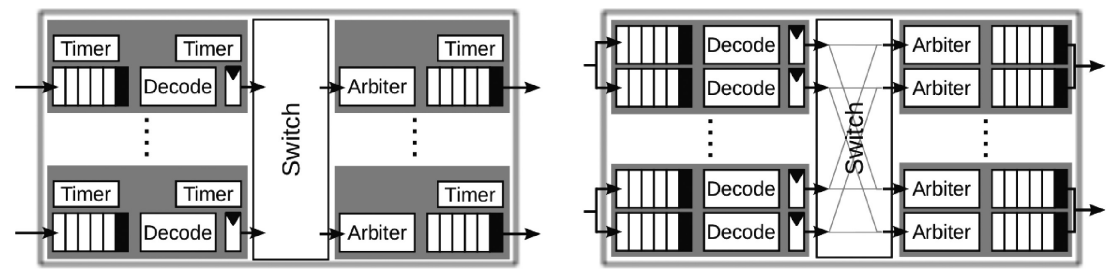

Fig. 4. Router architecture of a sdNoC router and a router implementing strict ordering.

\begin{tabular}{|c||c|c|}
\hline Queue length & sdNoC & Strict ordering \\
\hline 4 & 1.0 & 1.86 \\
\hline 8 & 1.70 & 3.23 \\
\hline
\end{tabular}

Fig. 5. Complexity of the router architectures relative to sdNoC with queue length of 4 .

freed transfer ids have to be used again. This is important, since it is possible that a retransmission is initiated shortly before the according acknowledgement packet arrives. In this case, a second acknowledgement packet could arrive for this transfer, assuming this transfer id would be already in use again, a different transfer would be acknowledged.

\section{HARDWARE COMPLEXITY}

\subsection{Routers}

The NoC routers are based on an input- and output-buffered architecture (see Figure 4), although the sdNoC concept is also applicable to routers that are only input buffered. The routers' decode logic (routing function) is implemented in an additional pipeline stage to enable higher clock rates. A timer, added to each FIFO in sdNoC routers, implements the criterion for discarding packets. In contrast, the routers based on strict ordering do not require timers, but have additional FIFO queues in each input and output port module. When using strict ordering a packet cannot change its virtual channel in the network. Consequently, the router's switch element is not a fully connected switch, but only connects virtual channels of the same group.

In order to compare the complexity of sdNoC routers to routers implementing strict ordering, we synthesized SystemVerilog models of both router architectures with a TSMC 65nm low power library. The fault tolerance features were omitted for this comparison, since we did not propose a measure for implementing fault tolerance together with strict ordering. Figure 5 shows the complexity of the two router architectures for different queue lengths of the input and output buffers. The complexity values are given relative to the $\mathrm{sdNoC}$ router equipped with queues that can store 4 flits. The complexity of the router with 4 flit queues applying strict ordering is $86 \%$ larger $\left(21,000 \mu \mathrm{m}^{2}\right)$ than the sdNoC router. This result is in line with our expectations, since the router applying strict ordering is equipped with twice the total buffer space compared to the sdNoC router. When comparing routers with the same total buffer space, that is, a router based on strict ordering with 4 flit queues and and an sdNoC router with 8 flit queues, strict ordering still leads to a $10 \%$ higher router complexity.

\subsection{Network Adapters}

The complexity evaluation of the network adapters (NA) is done in reference to a base architecture that comprises DMA functionality and a 10 flit long input queue. The DMA capabilities (included in the send manager in Figure 3(a)) enable the cores of a tile to offload sending and receiving flits to the NA. To obtain a network adapter 


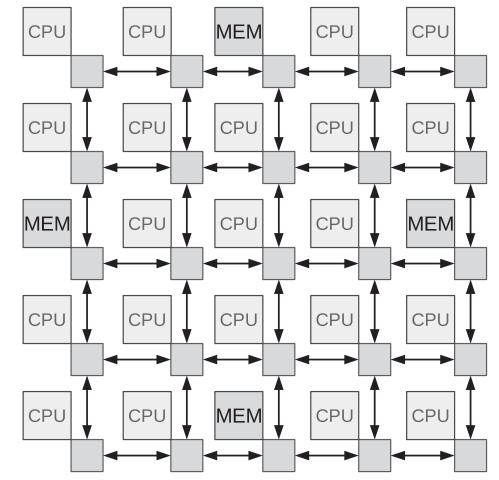

Fig. 6. Network architecture and mapping of tiles.

with retransmission capabilities for 4 packets, the FSM for sending has to be extended with 4 timers. This increases the area of the sending FSM by $11 \%\left(150 \mu \mathrm{m}^{2}\right)$, which is an increase of $1 \%$ of the total network adapter. If the retransmission buffer for the 4 packets is realized in the local memory of the tile, which is our favored architecture, 1360 bits have to be reserved there. This is only a very small share of the planned, typical sizes of such local memories (100KBit to 1MBit).

For the alternative architecture (shown in Figure 3(b)), in which the retransmission buffer is realized in the network adapter, four 10 flit output queues have to be added to the base network adapter. This approximately doubles the size of the base NA.

The complexity of the network adapter for strict ordering largely depends on the size of the input queues. Adding a second 10 flit long input queue to the base NA, results in $27 \%$ overhead. As an alternative, the 10 flit long input queue of the base NA could be halved, in order to obtain two queues for the virtual channels. Then, the NA for strict ordering would be approximately equal to the base NA.

In comparison, the savings per sdNoC router $\left(21,000 \mu \mathrm{m}^{2}\right)$ are much higher than the additional overhead in the network adapters $\left(150 \mu \mathrm{m}^{2}\right.$ for sending FSM; assuming retransmission buffer in the local memory). Obviously, the reason for the low overhead in the NA is that the DMA capabilities already provide much of the functionality required for retransmission. However, if the processor cores of the compute tiles would have to carry out the DMA functionality themselves, the software overhead would increase and the performance would decrease.

\section{EXPERIMENTS}

In the following our proposed $\mathrm{sdNoC}$ concept is evaluated in regard to solving the problem of message-dependent deadlocks. Therefore it is compared to a NoC that employs strict ordering realized by virtual channels which is currently a standard solution to tackle this problem. In addition, the dimensioning of key parameters of the bounded loss concept is presented. The simulation is carried out with a cycle accurate and highly configurable simulation model, which was developed at our institute. It is based on the network simulation framework OMNeT++.

For the investigation of message dependent deadlocks, the simulated SoCs use request-response traffic between processor and memory tiles to provoke the creation of cycles of packets. The systems consists of 4 memories located in the middle of each border of the mesh (see Figure 6), and 60 processor tiles. This network architecture is similar to the NoC of Tilera Tile64 [Wentzlaff et al. 2007]. All investigated networks are $8 \times 8$ meshes, employing wormhole forwarding and XY-routing. If not stated otherwise, the buffer length of input channel queues is 3 flits (including the register 

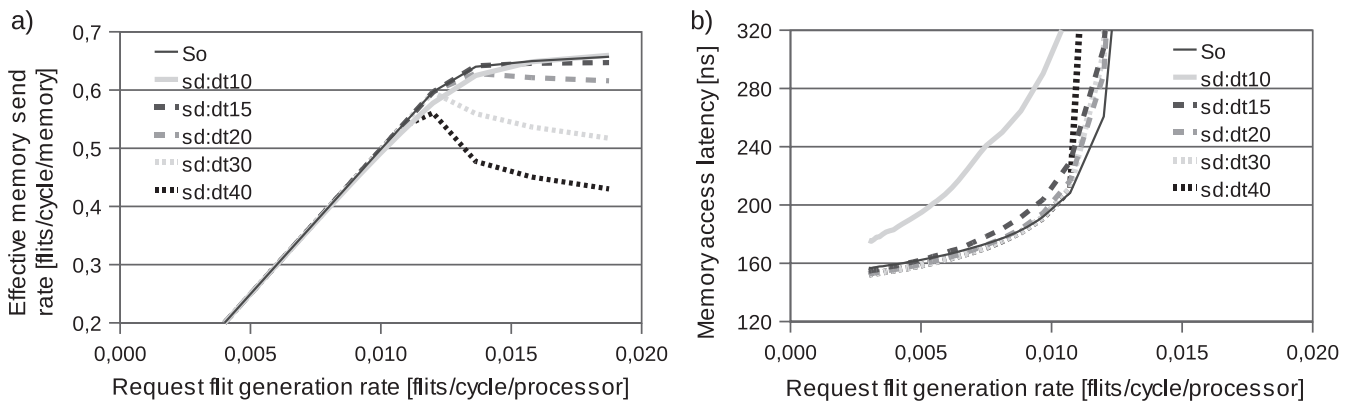

Fig. 7. Average effective memory send rate and memory access latency dependent on drop timings.
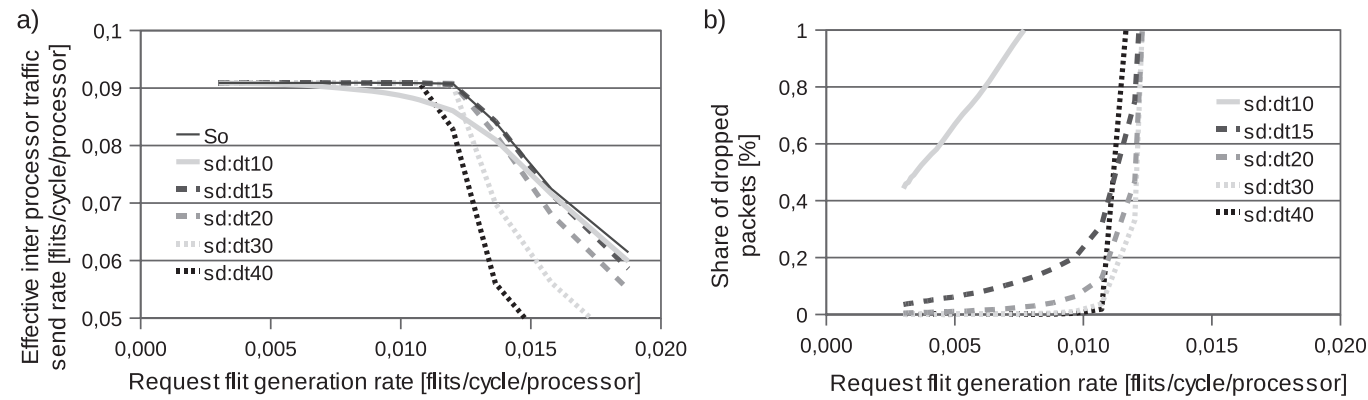

Fig. 8. Average effective send rate of inter processor traffic and share of dropped packets dependent on drop timings.

after the decode unit) and that of output buffer queues 2 flits. These are the minimum queue sizes to avoid stop and go of the transported flits. With 5 ports, input and output buffers, 2 virtual channels, this results in a total buffer size of 50 flits per router. If there were more dependent message types in the system, the minimum buffer size of the router applying strict ordering would be even higher. The sdNoC routers also use the minimum queue sizes of 3 flits for an input queue and 2 flits for an output queue. Since virtual channels are not required in $\mathrm{sdNoC}$ based networks, one router has only half the total buffer size ( 25 flits) compared to a router implementing strict ordering.

The applied traffic scenario consists of two different traffic classes. One class is inter processor traffic that serves as background traffic. Its destinations are uniformly distributed, the generation rate is set to 0.09 flits/cycle (see Figure 8(a)), which is approximately half the rate of the network's maximum throughput, and the packet size is 5 flits. The second class of traffic is the already mentioned memory access traffic, which consists of two dependent message types: read requests (packet size: 3 flits) and responses (packet size: 10 flits). The generation rate of the request flits is incremented over multiple simulations and the throughput or latency values of these simulations are used for the evaluation in the following graphs. As 60 processors are uniformly accessing the 4 memories the request rate per processor stays relatively low also when the network is already in saturation. In case of the $\mathrm{sdNoC}$ the effective send rate of the response flits is the actual send rate minus the dropped flits.

\subsection{Evaluation of Drop Timings}

One of the parameters that have a significant effect on the throughput of sdNoC is the condition for dropping a packet. The threshold value of our timer based criterion, is the drop timing dtX, with $\mathrm{X}$ being the number of cycles after which a packet is dropped if its header flit stays in the same queue. Therefore, in Figures 7 and 8 the 
NoCs that actively drop packets are denoted with sd:dtX. The retransmission period is set to 400 cycles and the number of outstanding requests per processor, that is, the retransmission buffer size, is 4 packets for the lossy NoCs in these simulations. The network with 2 virtual channels that applies strict ordering is called So.

From Figure 7(a), showing the effective send rate of the memories, and Figure 8(a), showing the send rate of interprocessor traffic, we can see that a drop timing of 15 cycles is the best value (sd:dt15). Due to the drop rate that stays below $0.1 \%$ when the network is not in saturation (Figure 8(b)), which is noticeable, but not too high, this network also provides low average latencies (see Figure 7(b)). However, the worst case latencies are higher than for So because of single packets that have to be retransmitted multiple times (up to 3 times in the simulations). Decreasing the drop timing to $10 \mathrm{~A}$ cycles as done with network sd:dt10 leads to an increase of the drop rate and a reduction of the effective network throughput. Although, sd:dt10 also reaches the peak memory throughput at higher request flit generation rates (0.018), the higher packet drop rate has to be paid with significantly higher latencies. The networks with higher drop timings (dt20, dt30, dt40) show not only a reduced share of dropped packets but also reduced throughput figures, because deadlocks or local congestions are not solved fast enough. This proves that our proposed approach of early discarding single packets leads to a higher effective network throughput. While for sd:dt20 this throughput reduction is relatively small compared to sd:dt15, for the networks with even higher drop timings the decrease in throughput is significant. The optimum drop timing is closely related to the length of the packets in the network, since the length of the packets determines how long they block other packets in the routers. Here, the majority of packets is 5 flits long and the maximum packet size is 10 flits. The difference between minimum and maximum packet sizes in the network should not be too high so that the duration of temporary blockages are relatively equal. Longer messages then have to be segmented into shorter packets, which also helps to reduce the probability of deadlocks.

In addition, to the determination of adequate drop timing, these simulations also show that the networks that actively drop packets can achieve an equal performance as the network applying strict ordering (So), with only half the buffer space in the network. Not only in terms of throughput (Figure 7(a), Figure 8(a)), but also in terms of average latency as Figure 7(b) shows. The reason is that the network So cannot use the whole buffer space in the network freely for all the packets currently in the network. Instead the assignment of the packets to the virtual channels depends on the message type (request, response) and thus is static.

\subsection{Investing Additional Buffer Space}

If we want to improve the network performance of the sdNoC by investing additional buffer space, the question is whether it would be more advantageous to just increase the router queue lengths or to introduce virtual channels in sdNoC. In contrast to the virtual channels in the network So ( 2 virtual channels, 3 flit input queues, 2 flit output queues), the virtual channels in sdNoC could be freely used independent of the message type. To answer this question, sdNoC based networks with and without virtual channels and different queue lengths were also simulated. As in the previous simulations, the number of outstanding requests is 4 , the retransmission period is 400 cycles and the drop timing is now set to 15 cycles for the networks based on sdNoC, which was shown before to be the best choice.

Figure 9(a) shows the send rate of response flits per memory for the networks. The network sd:2vc,i3o2, which is the lossy NoC with the same buffer configuration as the network So performs worse than the network sd:i5o5 that is just equipped with single 5 flit long input and output queues (sd:iXoY denotes the size of the input queues $\mathrm{X}$ and the output queues Y). sd:2ve,i3o2 performs even worse than So, which is due to 

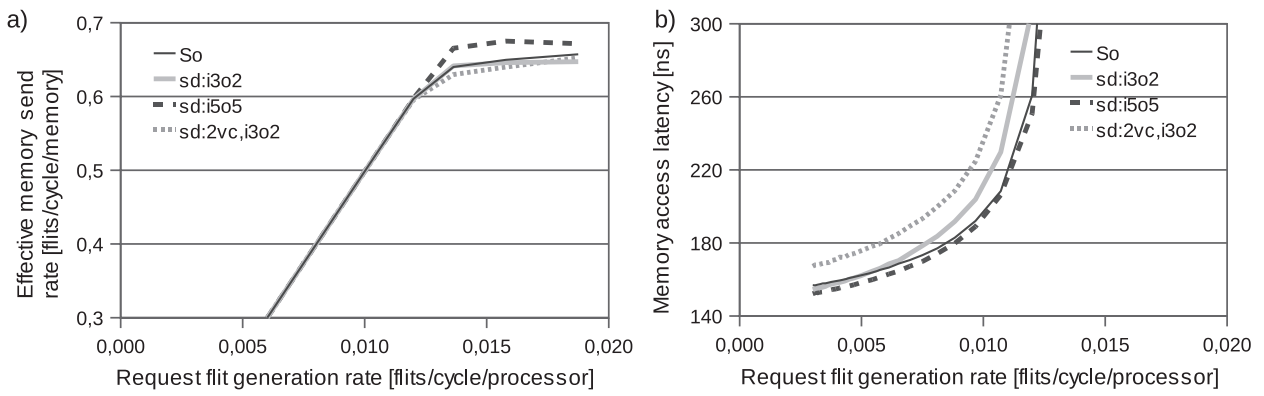

Fig. 9. Average effective memory send rate and memory access latency dependent on buffer configurations.
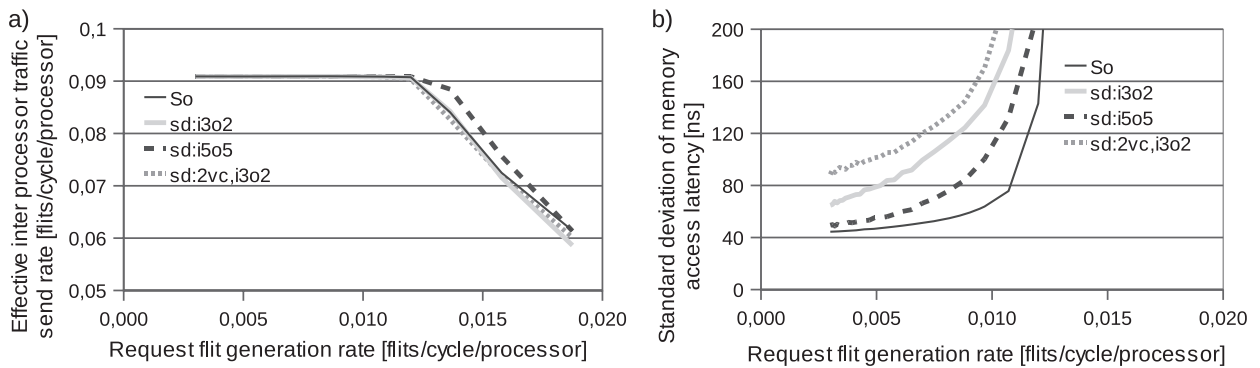

Fig. 10. Share of dropped packets and standard deviation of memory access latency dependent of buffer configurations.

the overhead in sdNoC caused by the acknowledging and retransmission. sd:2vc,i3o2 was simulated with drop timings of 10 cycles. Other timings and retransmission buffer sizes were also explored, but could not achieve better throughput.

From Figure 9(a) we can also see that the sdNoC based network with 2 virtual channels cannot improve its performance compared to the sd:i3o2, which uses only half the buffer space in the routers. While sd:2vc,i3o2 still shows comparable memory throughput, the memory access latency and the standard deviation of the memory access latencies are noticeably worse. In contrast, sd:i5o5, which has the same total buffer space, but simpler router architectures, outperforms all the other modeled networks. sd:i5o5 even shows better memory and inter processor traffic throughput (see Figure 10(a)) and a slightly better average memory latency (see Figure 9(b)) than So, only the standard deviation of the memory latencies shown in Figure 10(b) is higher, due to the retransmission of packets occurring in sdNoc.

In contrast to our expectations, these results show that a longer single buffer queue (up to a certain size) can be preferable over implementing additional virtual channels. One reason is that the timer based discard criterion does not work well for virtual channels, because packets can advance alternately in the different channels. Thus, the condition for discarding a packet will only become true in case of deadlocks and only rarely in case of temporary blockages. The second reason is related to the message dependencies between requests and responses, which leads to a congestion of the incoming requests at the memories. This back-pressure occurs at the sender interface, since the memories send out a 10 flit response for each received 3 flit request. While in general virtual channels can help to resolve congestions in a network, virtual channels cannot help with this kind of congestion. In case of such a back pressure of requests into the network, it turns out that longer router buffer queues are more advantageous than 2 virtual channels with half the queue length. However, if the message dependency 
a)

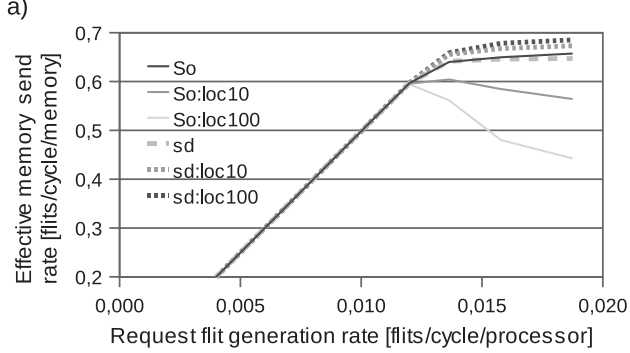

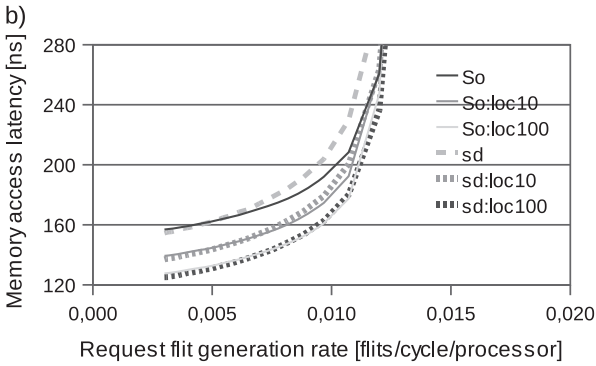

Fig. 11. Average effective send rate of memories and memory access latency dependent on localization of memory access traffic.

is omitted in the model (equal to an infinitely long input queue of the memory), the memory can simply receive all incoming requests and no congestion of requests occurs. Then, as expected, the sdNoC with 2 virtual channels shows better throughput than the architectures with only one channel.

\subsection{Localized Memory Access Traffic}

In the previous simulations, the processors accessed the four memories uniformly. However, assuming an optimized data mapping, these accesses would be localized, so that processors access nearer memories more frequently than the one farther away. This sub section presents the effect of the localization of the memory access traffic on the memory throughput of the NoCs, while the total request send rate stays constant. The distribution of the shares of packets sent to one of the four memories from a processor $p$ is calculated depending on the network distance $d(p, m)$ to the memory $m$ according to the following formula:

$$
\text { Share }(p, m)=\frac{1}{\sum_{i=1}^{4}\left(1+\frac{L}{d(p, i)+1}\right)^{2}} \cdot\left(1+\frac{L}{d(p, i)+1}\right)^{2} .
$$

Hereby, the localization factor $\mathrm{L}$ denotes the degree of the localization. Increasing this factor results in more packets being sent to the closer memories. The simulations are done for localization factors of 10 (loc10) and 100 (loc100). The inter processor traffic is not changed in these simulations. The configuration of the sdNoC based networks is as follows: 4 outstanding requests (i.e., retransmission buffers can store up to 4 packets), 400 cycles retransmission period and 15 cycles drop timing.

Figure 11(b) shows that the networks applying strict ordering (So:locx) only profit from the localization in terms of latency, while the memory throughput is reduced with increasing localization (see Figure 11(a)). This is due to increased local congestions around the memories. While the uniformly distributed requests result in a more equal traffic distribution over all routers of the network, the localized memory accesses and the static XY-routing algorithm lead to more traffic in the routers directly around the memories and a less traffic in the routers farther away. The routers with the increased load, then become the bottleneck and thus the reason for the reduced memory throughput.

However, Figure 11(a) also shows that sdNoC (sd:locx) does not suffer from the localization of access traffic, but clearly outperforms the NoCs based on deadlock avoidance that require double buffer space in the routers. Instead, the networks sd:locx are able to better handle the local congestions and thus are even able to profit from the localization not only in terms of latency but also in terms of throughput. 

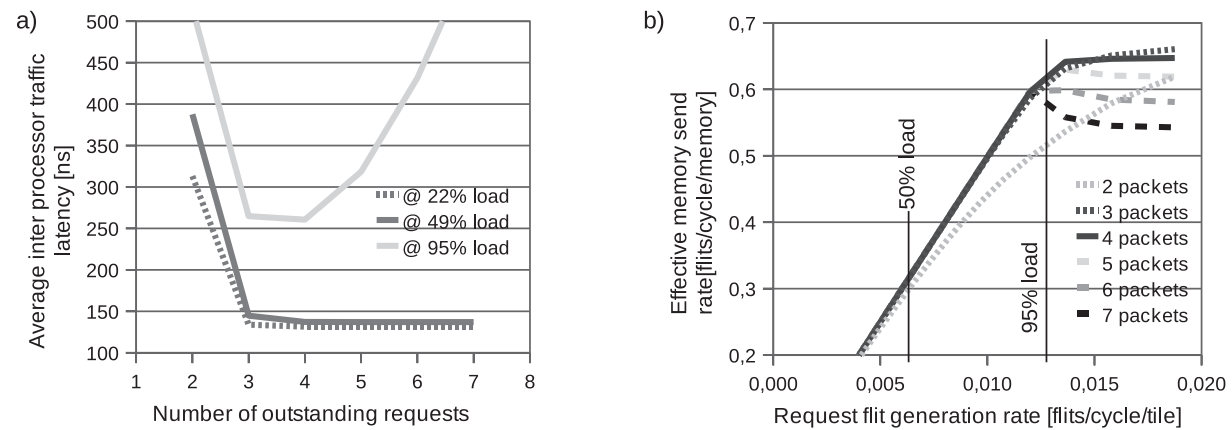

Fig. 12. Average inter processor latency and memory throughput depending on retransmission buffer size.

\subsection{Retransmission Buffer Size}

The retransmission buffer size that is, the number of outstanding requests has a direct effect on the sending bandwidth of a tile. If the number of possible outstanding requests is too low the send rate of the tile is limited by the retransmission buffer. In case of a too large retransmission buffer, the throughput is limited by the network, which gets constantly overloaded and thus drops more and more of the packets. Although in this case a higher number of packets is injected into the network, they do not reach their destination. Thus the effective throughput drops.

Figure 12 shows that the optimum number of outstanding requests for the modeled network architecture is four. Figure 12(a), depicting the transfer latencies of inter processor traffic for different load situations of the network, shows an increased average latency for small retransmission buffers. The load value is specified in \% of the maximum throughput of the network with retransmission buffers of 4 packets. The reduced memory throughput for small retransmission buffer sizes can be seen in Figure 12(b). It also shows that beyond the optimum size of the retransmission buffer of 4 packets, the effective network throughput is reduced and the transfer latency rises (see Figure 7(a)).

\subsection{Resend Period}

Another important parameter in our proposed concept is the retransmission period. This period should not be chosen too short, in order to allow a packet and its according acknowledgment packet to travel through the network despite of short temporary blockages. Consequently, the resend period depends on the distance between communication partners and thus on the size of the network. A too short timeout results in tiles resending their packets before the acknowledgement packets can reach the sending tile. In turn, this results in high traffic load in the network, which again leads to long transfer latencies and thus increases the probability for a resend. In the end, this leads to a reduced throughput as can be seen for the network with a 200 cycle period (sd:rp200) in Figure 13(a). The graphs of sd:rp400 and sd:rp600 in this figure also show that the throughput profits from higher retransmission values. However, higher retransmission periods have an effect on the transfer latency. This effect on the average latency is quite low, as long as the share of dropped packets is low (e.g., $0.1 \%$ like in the shown simulations). This is also proved by the good average latencies of the sdNoC based networks shown in the diagrams. However, the influence of high retransmission periods is visible in Figure 13(b), which shows the standard deviation of the latency. The retransmission value of 400 cycles, which was used for the networks in these simulations, which is a good trade-off. 

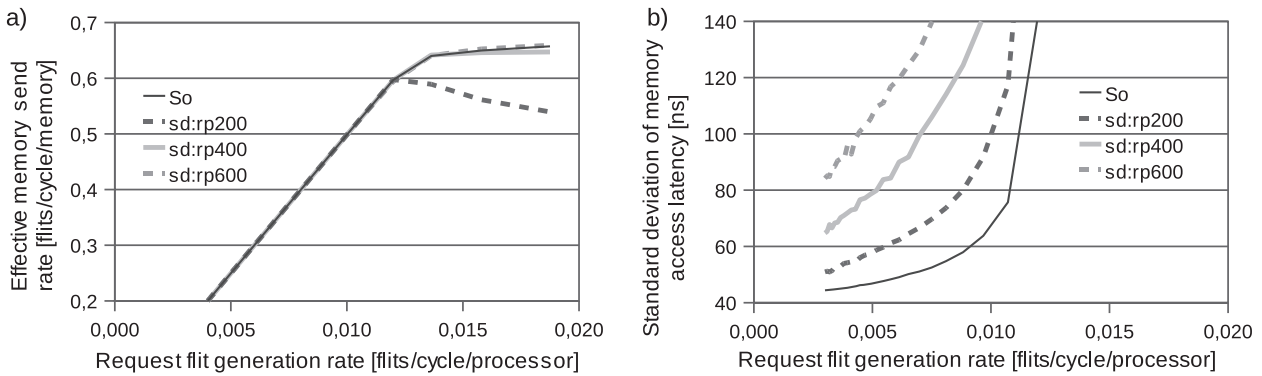

Fig. 13. Average effective send rate of memories and standard deviation of memory access latency dependent on retransmission period.
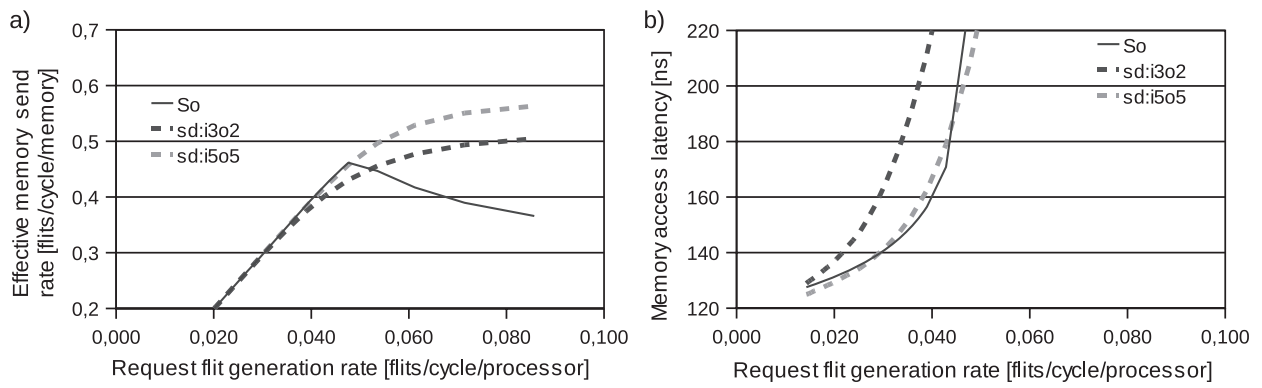

Fig. 14. Average effective memory send rate and memory access latency.
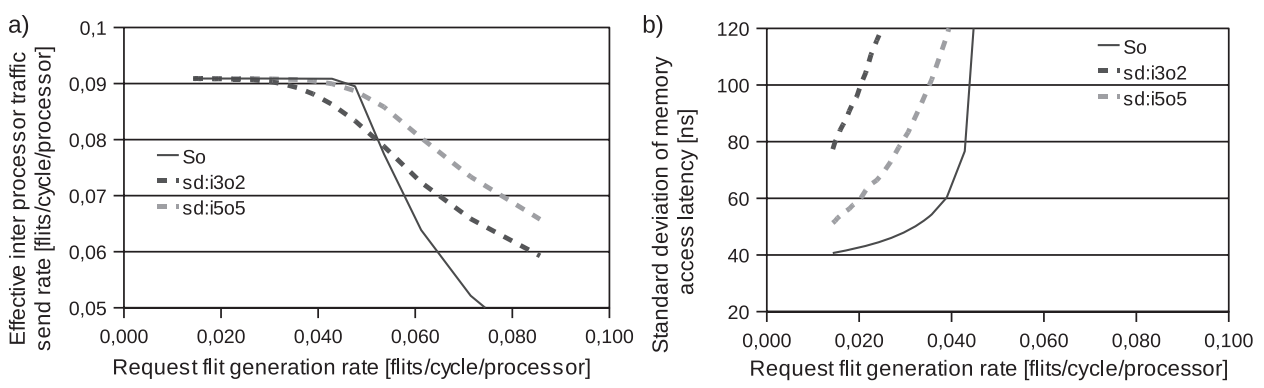

Fig. 15. Average throughput of inter processor traffic and standard deviation of memory access latency.

\subsection{Higher Memory Bandwidth}

The systems simulated in the previous simulations contained only 4 memories or memory interfaces, and thus provided only low memory access bandwidth. Systems that require higher memory bandwidth implement multiple memory interfaces or connect the memory interface to multiple routers at each border of the network. This section should show whether the sdNoC concept is also able to work in network architectures adapted for higher memory throughput. In contrast to the previous simulations, we now connect the memories not only to one router, but to the four routers at the middle of each border of the networks. Thus, the memories are now connected with four times the bandwidth than in the previous simulation. Note that Figure 14(a) still shows the effective send rate per connected link. To obtain the total memory throughput the given values would have to be multiplied by 16 . As in the simulations in section 5.3, the localization factor of the request traffic to the memories is 100.

The simulation results depicted in Figures 14 and 15 show that sd:i3o2, which operates with only half the buffer space of network So, can no longer compete. Although 

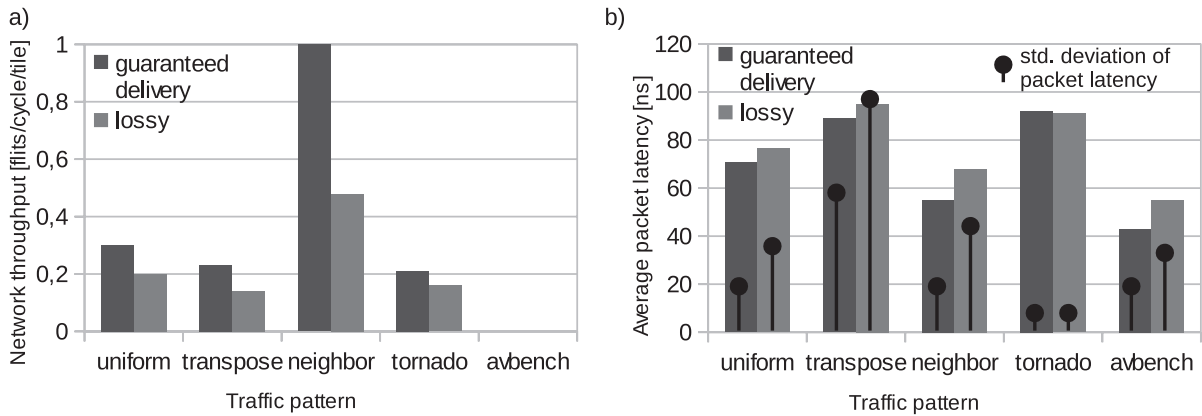

Fig. 16. Performance of standard guaranteed delivery $\mathrm{NoC}$ and $\mathrm{sdNoC}$ for different traffic patterns.

sd:i3o2 reaches high peak memory throughput as shown in Figure 14(a), this is only achieved by squeezing out the inter processor traffic (see Figure 15(a)). However, if equal buffer space is invested in the sdNoC routers, as done in sd:i5o5 in which input and output queues are extended to 5 flits, the lossy NoC can again compete with the network applying strict ordering. The average memory access latency of sd:i5o5 and of So shown in Figure 14(b) is almost similar, in terms of memory and inter processor traffic throughput the sdNoC based network is even better. As in the simulations before, the only drawback is the higher variance of the packet latencies originating from the retransmissions of packets. This can be seen in Figure 15(b), showing the standard deviation of the memory access latencies. While the buffer space in the networks is now equal, the sdNoC based network still has a simpler router architecture, and can provide fault tolerance due to its retransmission buffers.

\subsection{Basic Traffic Patterns and Audio Video Benchmark}

The simulations we have presented focus on the performance of networks in the presence of message dependent deadlocks. In contrast, the following simulations show the performance of the sdNoC compared to a standard guaranteed delivery NoC (no strict ordering) for traffic patterns, which are popular in the NoC community for comparing networks. This includes directed, synthetic traffic patterns like transpose, neighbor, and tornado traffic, that originate from matrix operations or fluid dynamics simulations [Dally and Towles 2003]. As before, the size of the simulated NoCs is $8 \times 8$, the input buffers of the routers are 3 flits long and the output buffers 2 flits. The packet length is set to 5 flits. Figure 16(a) shows that the throughput of sdNoC is lower for these traffic patterns than in a standard NoC. This is caused by the additional acknowledgement traffic in the lossy NoC. For the uniform traffic pattern the network sd:rp400,dt15 again showed the best performance. In case of traffic scenarios with lower hop counts like neighbor or tornado pattern, the best performance is provided for a shorter retransmission period (300 cycles). The throughput problem in case of the neighbor traffic originates from the huge difference in communication distance between the tiles in lowest row that send to the top row, and all the other tiles that only send to their diagonal neighbors 2 hops away. Figure 16(b) shows the average packet latencies at $50 \%$ of the maximum throughput of the according network (except avbench). While there is only a minor difference between the average latencies, the difference of the standard deviation of the packet latencies is significant in some of the scenarios.

Figure 16(b) also shows the performance of a realistic scenario, an audio video benchmark which was used in Hu et al. [2006] and in the meanwhile has been proposed as standard benchmark for NoCs. We manually mapped the benchmark's 16 tasks on $4 \times 4 \mathrm{NoCs}$ (running at $500 \mathrm{MHz}$, input buffers 3 flits long, output buffers 2 flits, 
5 flit packets). Hereby, we tried to keep communication distances at a minimum. Since this application consist only of directed traffic streams (data rate up to $1.2 \mathrm{GByte} / \mathrm{s}$ ), with low hop counts, only a few overlapping packet streams and no routing cycles due to dependent messages, the drop rate is very low at around $0,05 \%$. Therefore, the packet latencies (at full data rates of the benchmark) of the sdNoC (sd:rp200,dt15 with 4 packets per retransmission buffer) shown in Figure 16(b) are relatively independent of retransmission and drop timings. Nevertheless, a lower retransmission time gives best results, due to the low hop counts of the traffic. Compared to the guaranteed delivery NoC, sdNoC shows higher packet latencies due to the higher network load caused by the acknowledgements. For avbench, the throughput figures are not shown, since the data rates are predefined by the benchmark and not characteristic to the network.

\section{CONCLUSIONS AND OUTLOOK}

In this article we proposed sdNoC, a NoC concept that actively discards single packets in the network. This approach solves all types of deadlocks, and thus supersedes especially the costly solutions to handle message-dependent deadlocks. SdNoC inherently supports all communication protocols regardless of their number of dependent messages. The end-to-end retransmission scheme in the sdNoC concept provides lossless communication for the system's cores and reliable communication in the presence of transient errors. SdNoC not only secures the communication against bit flips in the transferred data, but also against any soft errors in the router logic. We showed that actively discarding packets also resolves temporary local congestions, and thus can increase the total effective network throughput.

Compared to strict ordering, sdNoC allows for simpler router architectures and better adaptability of the network architecture to the application's requirements. Buffer configurations in the sdNoCs routers, that is, virtual channels and queue lengths, are not determined by the message dependencies existing in the system, but can be chosen to either save chip area or to reach high throughput. In case of two dependent message types in the system, up to 50\% of chip area can be saved. If there are more dependent message types the savings can be even higher. The performance of sdNoC is strongly dependent on the traffic scenarios. For traffic patterns with hot spots and routing cycles (due to message dependencies) sdNoC can even outperform networks based on strict ordering in terms throughput and even average latency for some scenarios. Nevertheless, the worst case latencies are significantly higher using our approach. In case of traffic distributions like uniform, transpose, or neighbor traffic, which do not suffer from routing cycles or do not incorporate request response traffic, the network performance suffers from the additional acknowledge traffic. In order to enable the use of sdNoC also for latency sensitive applications, we will work on more sophisticated discard criteria in the future that try to avoid multiple retransmissions.

\section{REFERENCES}

Benini, L. ANd Micheli, G. D. 2002. Networks on chips: A new soc paradigm. Computer 35, 1, 70-78.

BJERREGAaRd, T. AND MAHADEVAN, S. 2006. A survey of research and practices of network-on-chip. ACM Comput. Surv. 38, 1, 1.

BoRKAR, S. 2005. Designing reliable systems from unreliable components: the challenges of transistor variability and degradation. IEEE Micro 25, 6, 10-16.

Borkar, S. 2010. The exascale challenge. In Proceedings of the International Symposium on VLSI Design Automation and Testing. 2-3.

Dally, W. and Towles, B. 2003. Principles and Practices of Interconnection Networks. Morgan Kaufmann Publishers Inc., San Francisco, CA.

Dally, W. J. AND Seitz, C. L. 1987. Deadlock-free message routing in multiprocessor interconnection networks. IEEE Trans. Comput. 36, 5, 547-553. 
Durand, Y., Bernard, C., ANd LattaRd, D. 2005. Faust: On-chip distributed architecture for a 4g baseband modem soc. In Design \& Reuse. IEEE Computer Society Press.

Fallin, C., Craik, C., ANd Mutlu, O. 2011. Chipper: A low-complexity bufferless deflection router. In Proceedings of the 17th IEEE International Symposium on High Performance Computer Architecture.

Floyd, S. AND JACOBSON, V. 1993. Random early detection gateways for congestion avoidance. IEEE/ACM Trans. Netw. 1, 397-413.

Frantz, A., Cassel, M., Kastensmidt, F., Cota, E., and Carro, L. 2007. Crosstalk- and seu-aware networks on chips. IEEE Des. Test Comput. 24, 4, 340-350.

Gebremichael-Tesfagiongis, B., Vaandrager, F. W., Zhang, M., Goossens, K., Rijpkema, E., Ruadulescu, A., Borrione, D., AND Paul, W. 2005. Deadlock prevention in sc thereal protocol. In Proceedings of the 13th IFIP WG Advanced Research Working Conference on Correct Hardware Design and Verification Methods.

Gomez, C., Gomez, M., Lopez, P., ANd Duato, J., D. 2008. BPS: A bufferless switching technique for NoCs. In Proceedings of the Workshop on Interconnection Network Architectures: 3rd International Conference on High-Performance Embedded Architectures and Compilers. 43-50.

Goossens, K., Dielissen, J., ANd Radulescu, A. 2005. Aethereal network on chip: Concepts, architectures, and implementations. IEEE Des. Test 22, 5, 414-421.

Gratz, P., Kim, C., McDonald, R., Keckler, S., and BuRger, D. 2006. Implementation and evaluation of on-chip network architectures. In Proceedings of the International Conference on Computer Design (ICCD'06). $477-484$.

Hansson, A., Goossens, A., AND Radulescu, A. 2007. Avoiding message-dependent deadlock in network-based systems on chip. VLSI Des. 10.

HaYenga, M., Jerger, N. E., AND Lipasti, M. 2009. Scarab: a single cycle adaptive routing and bufferless network. In Proceedings of the 42nd Annual IEEE / ACM International Symposium on Microarchitecture. ACM, New York, NY, 244-254.

Hoskote, Y., Vangal, S., Singh, A., Borkar, N., and Borkar, S. Sept.-Oct. 2007. A 5-gHz mesh interconnect for a teraflops processor. IEEE Micro 27, 5, 51-61.

Hu, J., OGRas, U. Y., and MARCULESCU, R. 2006. System-level buffer allocation for application-specific networkson-chip router design. IEEE Trans Comput.-Aid. Des. Integr. Circ. Syst. 25, 12, 2919-2933.

JANTSCH, A. AND Tehunen H. 2003. Networks on Chip. Kluwer, Hingham, MA.

KANG, Y. H., KWON, T.-J., AND DRAPER, J. 2010. Fault-tolerant flow control in on-chip networks. In Proceedings of the 4th ACM / IEEE International Symposium on Networks-on-Chip (NOCS'10). IEEE Computer Society, Los Alamitos, CA, 79-86.

Kim, J., Liv, Z., AND Chien, A. 1994. Compressionless routing: a framework for adaptive and fault-tolerant routing. In Proceedings of the 21st Annual International Symposium on Computer Architecture. 289-300.

LAI, M., WANG, Z., GAO, L., Lu, H., AND DAI, K. 2008. A dynamically allocated virtual channel architecture with congestion awareness for on-chip routers. In Proceedings of the 45th Annual Design Automation Conference (DAC'08). ACM, New York, NY, 630-633.

Lankes, A., Wild, T., Herkersdorf, A., Sonntag, S., And Reinig, H. 2010. Comparison of deadlock recovery and avoidance mechanisms to approach message dependent deadlocks in on-chip networks. In Proceedings of the 4th ACM/IEEE International Symposium on Networks-on-Chip. 17-24.

LiU, J. AND Delgado-Frias, J. 2006. A shared self-compacting buffer for network-on-chip systems. In Proceedings of the 49th IEEE International Midwest Symposium on Circuits and Systems (MWSCAS'06), Vol. 2. 26-30.

Mitra, S., Narayanan, V., Spainhower, L., And Xie, Y. 2005. Robust system design from unreliable components. Tech. rep., International Symposium on Computer Architecture.

Moscibroda, T. and Mutuu, O. 2009. A case for bufferless routing in on-chip networks. In Proceedings of the 36th Annual International Symposium on Computer Architecture (ISCA'09). ACM, New York, NY, 196-207.

Murali, S., Theocharides, T., Vijaykrishnan, N., Irwin, M. J., Benini, L., And Micheli, G. D. 2005. Analysis of error recovery schemes for networks on chips. IEEE Des. Test 22, 5, 434-442.

NeishabuRI, M. H. AND Zilic, Z. 2009. Reliability aware noc router architecture using input channel buffer sharing. In Proceedings of the 19th ACM Great Lakes Symposium on VLSI (GLSVLSI '09). ACM, New York, NY, 511-516.

Park, D., Nicopoulos, C., Kim, J., ViJaykRishnan, N., AND Das, C. R. 2006. Exploring fault-tolerant network-onchip architectures. In Proceedings of the International Conference on Dependable Systems and Networks (DSN '06). IEEE, Los Alamitos, CA, 93-104.

SaAstamoinen, I., Alho, M., AND Nurmi, J. 2003. Buffer implementation for proteo network-on-chip. In Proceedings of the International Symposium on Circuits and Systems (ISCAS'03). 
Song, Y. H. AND Pinkston, T. M. 2003. A progressive approach to handling message-dependent deadlock in parallel computer systems. IEEE Trans. Paral. Distrib. Syst. 14, 3, 259-275.

Su, W.-T., Shen, J.-S., And Hsiung, P.-A. 2011. Network-on-chip router design with buffer-stealing. In Proceedings of the 16th Asia and South Pacific Design Automation Conference (ASPDAC'11). IEEE Press, 160-164.

Wentzlaff, D., Griffin, P., Hoffmann, H., Bao, L., Edwards, B., Ramey, C., Mattina, M., Miao, C.-C., Brown III, J. F., AND Agarwal, A. Sept.-Oct. 2007. On-chip interconnection architecture ofthe tile processor. IEEE Micro 27, 5, 15-31.

Received July 2011; revised October 2011, December 2011; accepted March 2012 